\title{
Therapeutic Steroid Hormone
}

National Cancer Institute

\section{Source}

National Cancer Institute. Therapeutic Steroid Hormone. NCI Thesaurus. Code C1636.

Synthetically made hormones possessing the steroid ring system; e.g., androgens, estrogens, and adrenocortical hormones. 